\title{
Circulating angiostatin serum level in patients with systemic sclerosis
}

\author{
Zofia Gerlicz-Kowalczuk², Elzbieta Dziankowska-Zaborszczyk², Bożena Dziankowska-Bartkowiak³
}

${ }^{1}$ Department of Dermatology, Pediatric Dermatology and Dermatological Oncology, Lodz, Poland

2Department of Epidemiology and Biostatistics, the Chair of Social and Preventive Medicine of the Medical University of Lodz, Lodz, Poland ${ }^{3}$ Department of Dermatology and Venereology, Medical University of Lodz, Lodz, Poland

Adv Dermatol Allergol 2017; XXXIV (6): 543-546

DOI: https://doi.org/10.5114/ada.2017.72459

\begin{abstract}
Introduction: Systemic sclerosis (SSc) is achronic connective tissue disease characterized by microangiopathy with inadequate angiogenesis. Angiostatin (AS) is a potent antiangiogenic factor specifically inhibiting proliferation and inducing apoptosis of vascular endothelial cells.

Aim: To evaluate the level of angiostatin in the serum of patients with SSc.

Material and methods: Serum levels of AS were measured in 20 SSc patients and 12 healthy controls.

Results: A statistically significant difference in the serum levels of AS in SSc patients was observed compared to the control group ( $636.51 \mathrm{vs.} 869.20 \mathrm{ng} / \mathrm{ml} ; p=0.012$ ). Significant correlations between limited and disseminated SSc (ISSc/dSSc) were not found, however, a difference between ISSc and the control group was demonstrated (620.00 vs. $869.20 \mathrm{ng} / \mathrm{ml} ; p=0.011$ ). The serum level of AS was not associated positively with organ changes caused by SSc. However, a statistically significant lower serum level of AS was observed in patients with SSc and no esophageal $(p=0.008)$ or pulmonary changes $(p=0.007)$ compared to the control group.

Conclusions: Our results reveal significant differences in AS level in SSc patients compared to the healthy controls, and suggest that a low level of AS may occur as a result of impaired angiogenesis.
\end{abstract}

Key words: systemic sclerosis, angiostatin, angiogenesis, microangiopathy.

\section{Introduction}

Angiostatin (AS) is a proteolytic fragment of plasminogen and angiogenesis inhibitor [1]. Angiostatin shows specific activity towards endothelium vascular cells and directly stimulates their apoptosis and selectively stimulates their further proliferation [2]. Claesson-Welsh et al. demonstrated that AS acts through kinases focal adhesion kinase (FAK) [2]. The mechanism of the anti-angiogenic effect involves stimulating improper signals, which disturb proper function of junctions between endothelial cells and induces apoptosis.

Although the exact mechanisms by which AS inhibits angiogenesis remain unclear, Moser el al. found that AS binds ATP synthase on endothelial cell surface, which inhibits activity of the enzyme [3]. Thus, the intracellular concentration of ATP increases. In hypoxia, as observed in Raynaud's phenomenon, ATP is responsible for survival of endothelial cells. However, inhibition of ATP synthesis by AS leads to metabolic changes followed by reduction in proliferation and development of apoptosis cascade. An- giostatin also shows inhibitory activity of proangiogenic factors - basic fibroblast growth factor (bFGF) and vascular endothelial growth factor (VEGF) - on target cells by inducing transient dephosphorylation in endothelial cells of small blood vessels [4].

Systemic sclerosis (SSc) is a connective tissue disease characterized by microvascular regression due to endothelial dysfunction with immune system abnormalities [5-7]. Microangiopathy seems to be directly responsible for clinical manifestation.

\section{Aim}

The aim of the study was to evaluate the serum level of angiostatin, which may play a role in the progression of SSc.

\section{Material and methods}

Blood samples were collected from 20 SSc patients (19 females and 1 male; 16 limited SSc (ISSc), 4 diffuse

Address for correspondence: Zofia Gerlicz-Kowalczuk, Department of Psychodermatology, Medical University of Lodz, 251 Pomorska St build. C5, 92-213 Lodz, Poland, phone: +48 606168 467, e-mail: zosia_gerlicz@yahoo.com Received: 1.07.2016, accepted: 8.11.2016. 
SSc (dSSc) according to the criteria of LeRoy [7]) and 12 healthy individuals. All SSc patients fulfilled the criteria of the American College of Rheumatology [8] and EULAR [9]. The control group consisted of 12 randomly selected healthy subjects with no systemic diseases or on medication. All samples were taken between 7:00 and 9:00 a.m. The samples were centrifuged and the obtained sera were stored in aliquots at $-20^{\circ} \mathrm{C}$ until analyses. Clinical, laboratory and treatment data were collected at the time when the blood samples were drawn. Drugs, including corticosteroids and immunosuppressants, were authorized but stopped $24 \mathrm{~h}$ before blood collection. The patient characteristics are presented in Figure 1.

Approval for the study was obtained from the Medical University of Lodz (approval no. KE/3139/15 \& RNN/218/09/KE).

\section{Clinical assessment}

All extensive clinical parameters were established for each SSc patient. The history and complete physical examination were obtained from each patient. The patients were evaluated for the cardiac (diagnosed by Holter, ECG, echocardiography and cardiological consultation), pulmonary (chest RTG, high-resolution computed tomography (HRCT) scan of thorax and pulmonary consultation, if necessary), esophageal (esophageal scintigraphy), renal and hematological (blood test, bone marrow biopsy if needed) involvement, as described previously [10] (Figure 1).

\section{ELISA for angiostatin}

Levels of soluble Angiostatin were determined by $\mathrm{Hu}$ man Angiostatin ELISA Kit (RayBio ${ }^{\oplus}$, Norcross, Georgia, USA) strictly according to the manufacturer's instructions. Serum concentrations were calculated using a standard curve generated with specific standards provided by the manufacturer.

\section{Results}

A statistically significant difference in the serum level of AS between SSc patients and the control group was observed (636.51 vs. $869.20 \mathrm{ng} / \mathrm{ml} ; p=0.012$ ). Significant difference between limited and disseminated SSc patients (ISSc/dSSc) (620.00 vs. $702.53 \mathrm{ng} / \mathrm{ml} ; p>0.05$ ) were not found, however, the group of patients with dSSC was not representative. Differences between ISSC patients and the control group were observed (620.00 vs. $869.20 \mathrm{ng} / \mathrm{ml} ; p=0.011$ ) (Table 1).

In the AS serum level, there was a statistically significant difference between SSc patients with esophagus $(p=0.008)$ and pulmonary changes $(p=0.007)$ compared to the healthy group. There was significant difference ( $p=$ 0.013 ) between the patients with arthritis and the control group. Interestingly, the presented symptoms also showed a statistically significant difference between SSc patients with no headache $(p=0.01)$, no palpitation $(p=0.01)$, no dyspnea at rest $(p=0.01)$, no dyspnea on exertion ( $p=$ $0.01)$, no telangiectasia $(p=0.01)$.

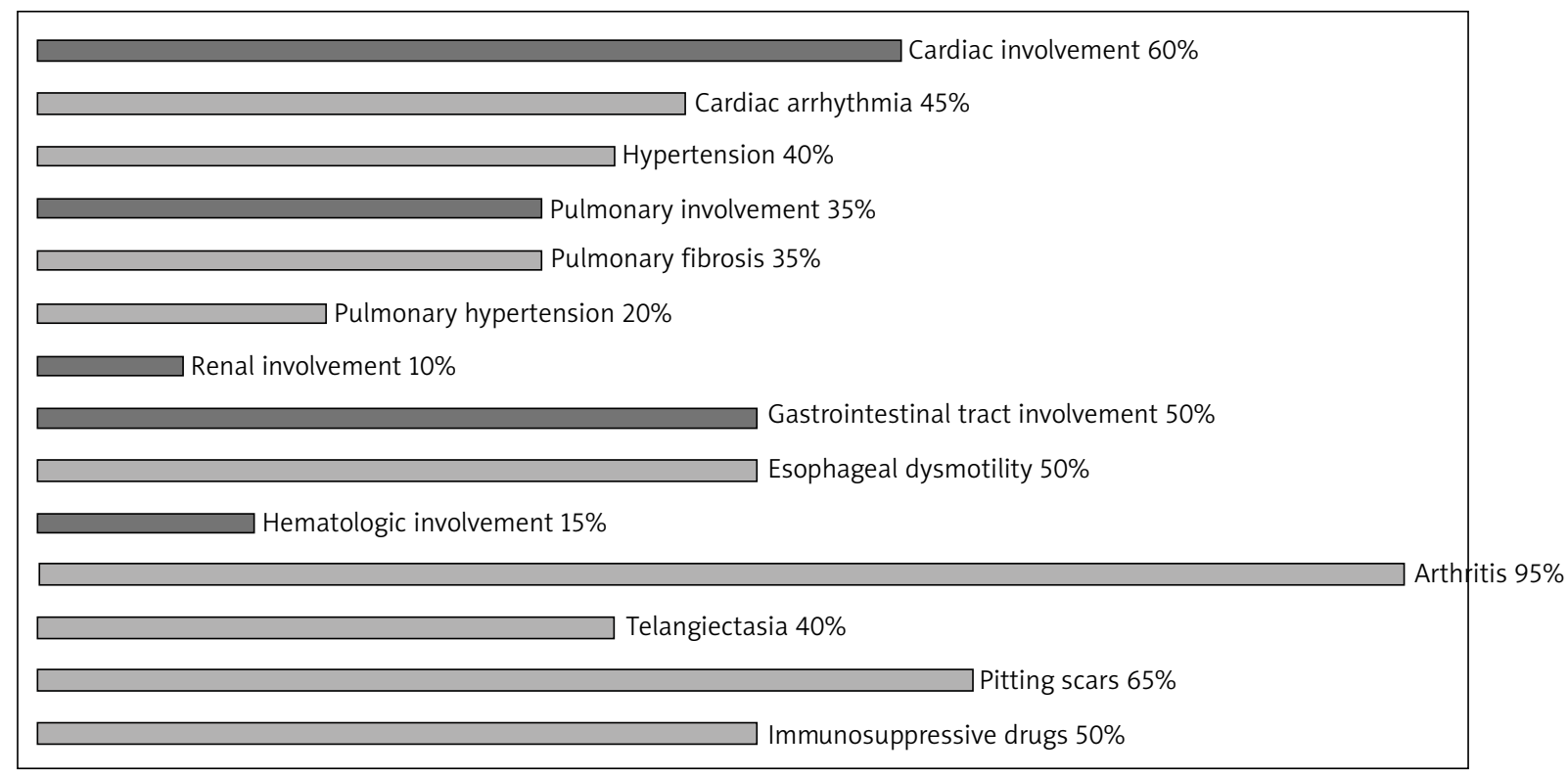

Figure 1. Clinical characteristics of SSc patients 
Table 1. Serum level of AS in SSc, ISSc, dSSc and control groups

\begin{tabular}{ccccc}
\hline $\begin{array}{c}\text { Angiostatin } \\
{[\mathrm{ng} / \mathrm{ml}]}\end{array}$ & SSc & ISSc & dSSc & Control group \\
\hline $\bar{x}$ & $636.51^{*}$ & 620.00 & $702.53^{*}$ & 869.20 \\
\hline Min.-max. & $128.66-922.50$ & $128.66-887.24$ & $465.8-922.50$ & $597.81-1176.00$ \\
\hline SD & 244.28 & 250.56 & 238.25 & 170.46 \\
\hline $\bar{x}-$ mean, SD - standard deviation, ${ }^{*}$ statistical significance $p<0.05$ vs. control group. & &
\end{tabular}

\section{Discussion}

In systemic sclerosis, inhibition of angiogenesis is of vital importance. Formation of new blood vessels results from synchronization of many pathways. Hirigoyen et al. demonstrated that platelets have an important role in progression of peripheral microvascular damage and ineffective vascular repair in patients with SSc [11]. Indeed, platelets are also active in the process, they release many angiogenic factors from $\alpha$-granulocytes, including two antagonists - VEGF and AS [12]. Although AS, compared to VEGF, is released with a delayed effect by karyotypes. Angiostatin is a fragment of plasminogen, inhibits proliferation and migration of epithelial cells, at the same time, promotes apoptosis of these cells [2].

In patients with SSc, Mulligan-Kehoe et al. found a significant decrease in plasmin activity and a high level of angiostatin at the same time, which stays in opposition to our findings [13]. The authors also focused on the plasminogen structure. It is known that plasminogen is produced in two forms, glutamic acid - plasminogen and lysine - plasminogen. Patients with SSc, compared to the healthy individuals, showed a significantly higher concentration of glutamic acid - plasminogen in plasma as well as a surprisingly low concentration of lysine - plasminogen [14]. Mulligan-Kehoe et al. also demonstrated that exposure of microvascular endothelial cells from healthy volunteers to plasma from patients with SSC showed lower ability in migration and angiogenesis compared to the cells exposed to the plasma from healthy individuals [14]. Also Almeida et al. observed an elevated serum level of AS along with endostatin in SSc patients. Moreover, organ involvement analysis revealed a correlation between osteoarticular involvement and the AS level [13]. Similar observations were published by Borghini et al., who observed that levels of the angiostatic mediators including angiostatin were all significantly elevated in sera from SSc patients compared with sera from both healthy controls and cyclophosphamide-treated SSc patients [15]. In our analysis, we did not observe any difference in the serum AS level between patients on immunosuppressive therapy and those who were not, however, we did not divide patients by the drug they take.

On the contrary, our results showed a low serum AS concentration in patients with SSc, which may be related to positive feedback between impaired angiogenesis due to the disease and AS. Under hypoxic conditions, VEGF signaling stimulates angiogenesis while the increased angiostatin level would promote endothelial cell death. A decreased level of AS, as observed in our SSc patients, is in accordance with proper response to insufficient angiogenesis. It is confirmed by the fact that patients with a low concentration of AS did not develop esophagus or pulmonary changes and reported fewer symptoms.

\section{Conclusions}

Decreased AS may contribute to inhibition of microangiopathy through normalization of endothelial cellmatrix interactions, as well as reduction of endothelial cell apoptosis. Based on the obtained data, we concluded that low level of angiostatin may play a preventive role in organ involvement in SSc.

\section{Acknowledgments}

The study was supported by the Medical University of Lodz No. 503/1-137-04/503-11-001, 502-03/1-137-04/50214-205 and 503/1-152-01/503-01.

\section{Conflict of interest}

The authors declare no conflict of interest.

\section{References}

1. O'Reilly MS, Holmgren L, Shing Y, et al. Angiostatin: a novel angiogenesis inhibitor that mediates the suppression of metastases by Lewis lung carcinoma. Cell 1994; 79: 315-28.

2. Claesson-Welsh L, Welsh M, Ito N, et al. Angiostatin induces endothelial cell apoptosis and activation of focal adhesion kinase independently of the integrin-binding motif RGD. Proc Natl Acad Sci USA 1998; 95: 5579-83.

3. Moser TL, Stack MS, Asplin I, et al. Angiostatin binds ATP synthase on the surface of human endothelial cells. Proc Natl Acad Sci USA 1999; 96: 2811-6.

4. Redlitz A, Daum G, Sage EH. Angiostatin diminishes activation of the mitogen-activated protein kinases ERK-1 and ERK-2 in human dermal microvascular endothelial cells. J Vasc Res 1999; 36: 28-34.

5. Nicolosi PA, Tombetti E, Maugeri N, et al. Vascular remodelling and mesenchymal transition in systemic sclerosis. Stem Cells Int 2016; 2016: 4636859. 
6. Manetti M, Guiducci S, Ibba-Manneschi L, et al. Mechanisms in the loss of capillaries in systemic sclerosis: angiogenesis versus vasculogenesis. J Cell Mol Med 2010; 14: 1241-54.

7. LeRoy EC, Black C, Fleischmajer R, et al. Scleroderma (systemic sclerosis): classification, subsets and pathogenesis. J Rheumatol 1988; 15: 202-5.

8. Masy AT, Rodnan GP, Medsger TA, et al. Diagnostic and Therapeutic Criteria Committee (1980). Preliminary criteria for the classification of systemic sclerosis (scleroderma). Arthritis Rheum 1980; 23: 581-90.

9. van den Hoogen F, Khanna D, Fransen J, et al. 2013 classificaation criteria for systemic sclerosis: an American College of Rheumatology/European League against Rheumatism collaborative initiative. Arthritis Rheum 2013; 65: 2737-47.

10. Gerlicz Z, Dziankowska-Bartkowiak B, Dziankowska-Zaborszczyk E, et al. Disturbed balance between serum levels of receptor tyrosine kinases Tie-1, Tie-2 and angiopoietins in systemic sclerosis. Dermatology 2014; 228: 233-9.

11. Hirigoyen D, Burgos PI, Mezzano V, et al. Inhibition of angiogenesis by platelets in systemic sclerosis patients. Arthritis Res Ther 2015; 17: 332.

12. Radziwon-Balicka A, Moncada de la Rosa C, Jurasz P. Plateletassociated angiogenesis regulating factors: a pharmacological perspective. Can J Physiol Pharmacol 2012; 90: 679-88.

13. Almeida I, Oliveira Gomes A, Lima M, et al. Different contributions of angiostatin and endostatin in angiogenesis impairment in systemic sclerosis: a cohort study. Clin Exp Rheumatol 2016; 34 (Suppl 100): PI 0037-PF 0042.

14. Mulligan-Kehoe MJ, Drinane MC, Mollmark J, et al. Antiangiogenic plasma activity in patients with systemic sclerosis. Arthritis Rheum 2007; 56: 3448-58.

15. Borghini A, Manetti M, Nacci F, et al. Systemic sclerosis sera impair angiogenic performance of dermal microvascular endothelial cells: therapeutic implications of cyclophosphamide. PLoS One 2015; 10: e0130166. 